\title{
Electric field control of spin-orbit interaction and superconductivity at oxide interfaces
}

1. Tunable Rashba spin-orbit interaction at oxide interfaces, N. Reyren, S. Thiel, A.D. Caviglia, M. Gabay, S. Gariglio, N. Reyren, C. Cancellieri, and J.-M. Triscone, arXiv:0912.3731.

2. Tuning spin-orbit coupling and superconductivity at the $\mathrm{SrTiO}_{3} / \mathrm{LaAlO}_{3}$ interface: a magneto-transport study, M. Ben Shalom, M. Sachs, D. Rakhmilevitch, A. Palevski, and Y. Dagan, arXiv:1001.0781.

\section{Recommended with a Commentary by Atsushi Fujimori, University of Tokyo}

Control of electron spins by electric field is a major goal of spintronics since applying electric field to electronic devices in a controlled manner is much easier and more practical than applying magnetic field. In their seminal paper [1], Datta and Das proposed a device in which the spins of electrons transported through a semiconductor channel between two ferromagnetic electrodes can be rotated if a gate voltage is applied perpendicular to the current. The origin of the rotation is an effective magnetic field $\mathbf{B}_{\text {eff }} \propto \mathbf{E} \times \mathbf{k}$, where $\mathbf{E}$ is the applied electric field, and $\mathbf{k}$ is the electron momentum. The Zeeman splitting caused by $\mathbf{B}_{\text {eff }}$ is called Rashba effect or Rashba spin-orbit splitting.

Recently, much attention has been paid to oxide interfaces with "polarity discontinuity" such as $\mathrm{LaAlO}_{3} / \mathrm{SrTiO}_{3}(001)(\mathrm{LAO} / \mathrm{STO})$ because of the surprising occurrence of high mobility 2D electron gas (2DEG) [2] and even its superconductivity [3]. Such a polarity discontinuity produces an intense electric field perpendicular to the interface and, therefore, the 2DEG should experience Rashba effect if the electrons have finite spin-orbit interaction. In fact, the bottom of the conduction band of STO, where the 2DEG is formed, is originated from the three-fold degenerate $t_{2 g}$ orbitals of Ti $3 d$ and therefore should exhibit spin-orbit splitting (of order meV) [4]. This is a small energy but not negligible compared to the tiny Fermi energy of the dilute 2DEG and larger than the superconducting gap (of order $10 \mu \mathrm{V}$ ).

The recommended two papers report on experimental evidence for the effect of Rashba-type spin-orbit interaction on the magneto-transport of the 2DEG confined to the LAO/STO interface. In order to modulate the electric field and hence the $2 \mathrm{D}$ carrier density, gate voltage was applied perpendicular to the interface. The gate voltage dependence of magnetoresistance was attributed to the gate voltage dependence of Rashba interaction strength. From both papers, it was revealed that, when the system approaches the superconducting region through the gate voltage control both from the "overdoped" and "underdoped" sides ${ }^{1}$ Rashba spin-orbit interaction is enhanced. This indicates that Rashba effect favors the superconductivity at the interfaces, at least through the suppression of weak localization on the underdoped

\footnotetext{
${ }^{1} \mathrm{Bulk} \mathrm{SrTiO}_{3}$ crystals doped with electrons show a superconducting "dome" as a function of carrier density, from $8.5 \times 10^{18} \mathrm{~cm}^{-3}$ to $3.0 \times 10^{20} \mathrm{~cm}^{-3}$ with the maximum $T_{c}$ of $300 \mathrm{mK}$, like the high $-T_{c}$ cuprates, and so does the LAO/STO interface as a function of gate voltage with nearly the same maximum $T_{c}$.
} 
side. Moreover, because of the inversion symmetry breaking at the interface, the superconductivity may not be a simple BCS-type $s$-wave as in bulk $\mathrm{SrTiO}_{3}$, but may be unconventional as in the case of heavy-Fermion superconductor without inversion symmetry $\mathrm{CePtSi}_{3}$ [5]. If the superconductivity is unconventional, an unknown mechanism by which the spin-orbit interaction enhances the superconductivity might be present.

Apart from the superconductivity, magnetism of the LAO/STO interface was reported to be complex: Field-induced magnetism [6] and ferromagnetism [7] would have to be re-considered under the influence of Rashba spin-orbit interaction. In order to understand why the strength of Rashba interaction depends so dramatically on the gate voltage, the electric field dependence of the spatial extent of the electron wavefunction should be considered, as pointed out by a recent magneto-transport study of the LAO/STO interface [8]. In that study, it was shown that the gate voltage controls the mobility rather than the density of the 2DEG through the modification of the wavefunction. This suggests that structural disorder at the interfaces is a more important ingredient of the interesting electronic properties of oxide interfaces than previously thought.

[1] S. Datta and B. Das, Appl. Phys. Lett. 56, 665 (1990).

[2] A. Ohtomo and H. Y. Hwang, Nature 427, 423 (2004).

[3] N. Reyren, S. Thiel, A.D. Caviglia, L.F. Kourkoutis, G. Hammerl, C. Richter, C.W. Schneider, T. Kopp, A.-S. Ruetschi, D. Jaccard, M. Gabay, D. A. Muller, J.-M. Triscone, and J. Mannhart, Science 317, 1196 (2007).

[4] L.F. Mattheiss, Phys. Rev. B 6, 4718 (1972).

[5] E. Bauer, H. Kaldarar, A. Prokofiev, E. Royanian, A. Amato, J. Sereni, W, Bramer-Escamilla, and I. Bonalde, J. Phys. Soc. Jpn. 76, 051009 (2007).

[6] S. Seri and L. Klein, Phys. Rev. B 80, 180410(R) (2009)

[7]A. Brinkman, M. Huijben, M. Van Zalk, J. Huijben, U. Zeitler, J.C. Maan, W.G. Van Der Wiel, G. Rijnders, D. H. A. Blank, and H. Hilgenkamp, Nature Mater. 6, 493 (2007).

[8] C. Bell, S. Harashima, Y. Kozuka, M. Kim, B.G. Kim, Y. Hikita, and H. Y. Hwang, Phys. Rev. Lett. 103, 226802 (2009) 